\title{
Magnetic Resonance Imaging for Screening of Woman at High-Risk of Breast Cancer
}

\author{
Reham G. Garout, Howayda M. Ahmed, Saddig D. Jastaniah*, Ibrahim A. Awad \\ Department of Diagnostic Radiology, Faculty of Applied Medical Sciences, King Abdulaziz University, Jeddah, \\ KSA \\ Email: sjastaniah@kau.edu.sa
}

Received 10 May 2014; revised 6 June 2014; accepted 13 June 2014

Copyright @ 2014 by authors and Scientific Research Publishing Inc.

This work is licensed under the Creative Commons Attribution International License (CC BY).

http://creativecommons.org/licenses/by/4.0/

(c) (i) Open Access

\begin{abstract}
MRI is an excellent option for detection of breast cancer for some selected groups, including those patients with a high probability to hit the disease. However, the high costs and low availability of the device have led to a decline in the application of imaging MRI. The aim of this study was to review usefulness of MRI as a new complementary way to detect breast cancer in routine annual checkup for women breasts of certain ages and breast mass. A cross-sectional Descriptive MRI study was performed on 105 asymptomatic women with a mean age of 49 years. The study group with at least one risk factor of breast cancer were presenting for routine annual screening or follow up at King Abdulaziz University Hospital in Jeddah. It has been found that, 48 patients had biopsy, they were recommended by magnetic resonance imaging and only 14 had positive results, while magnetic resonance imaging suggested 16 and mammography had 62 positive results. Magnetic resonance imaging is not recommended for the average-risk or the general population either; it had been advised for screening the high-risk women of breast cancer. Sensitivity of magnetic resonance imaging has been found to be much higher than of mammography but specificity was generally lower. We propose that it is reasonable to consider MRI as a complement to mammography in screening patients who were at high risk for breast cancer because Magnetic Resonance Imaging can detect small foci that are occult in mammography but we don't advise to check with the general population.
\end{abstract}

\section{Keywords}

MRI, Mammogram, Breast Cancer, Women Breasts

\footnotetext{
${ }^{*}$ Corresponding author.
}

How to cite this paper: Garout, R.G., Ahmed, H.M., Jastaniah, S.D. and Awad, I.A. (2014) Magnetic Resonance Imaging for Screening of Woman at High-Risk of Breast Cancer. Advances in Breast Cancer Research, 3, 59-67. 


\section{Introduction}

Every year, over a million women worldwide are diagnosed for breast cancer. The best chance for cure is offered by early detection. In average-risk women, mammogram is an effective method for early detection. However, its sensitivity tends to decrease in women at high risk for breast cancer. Because of its better sensitivity over mammogram, multiple investigators have studied the potential role of MRI in screening women at high risk. [1]. Hence, in many cases, the patient may be asked to undergo magnetic resonance imaging [2] [3].

Breast MRI is very sensitive and is useful for assessing invasive carcinomas. It is also used to assess high-risk patients who have more than a 20 percent chance of developing breast cancer in their lifetimes based on genetics (BRCA1 and BRCA2) and strong family history of breast cancer. If a MRI detects a suspicious lesion that isn't recognizable on the mammogram, then a second-look ultrasound is recommended. If visible, the lesion may be biopsied under ultrasound guidance; if not, it may need to be biopsied under MRI guidance [4].

MRI was first widely used in the 1990s to find ruptured breast implants. It began to turn up tumors in dense breast tissue after gadolinium. Since then, MRI with intravenous contrast agent has been used as follow up test for suspicious mammograms. Only in the last few years clinical result suggested that MRI may have role as screening tool for early detection of breast cancer [5]. However, MRI is still considered an investigational technique for surveillance and screening of asymptomatic women with normal conventional imaging findings.

The aim of this study was to demonstrate effectiveness, usefulness and limitations of MRI as a new complementary tool for breast cancer screening of high risk populations at certain ages and breast masses.

\section{Materials and Methods}

\subsection{Study Design}

This study was done in the department of diagnostic radiology, King Abdul-Aziz University Hospital situated in Jeddah city, Kingdom of Saudi Arabia (KSA). A cross-sectional observational study was performed during the academic year 2012-2013, after obtaining research ethical approval.

\subsection{Patients}

The study was performed on women who were asymptomatic presenting for routine annual screening or follow up with at least one risk factor for breast cancer. The collected data was 105 patients ranging in age from 26 to 81 years old with the mean age is 48.94 years and Standard deviation 10.83. Age had been categorized as shown in Table 1.

\subsection{Methods}

Breast MRI was performed using MAGNETOM Version 3 T Siemens machine with the 16-Channel Breast array Coil. Post contrast study was done using a Dotarem contrast with concentration 0.5 and the dose $0.2 \mathrm{ml}$ per kg.

\subsection{Patient Preparation}

Intravenous lines were introduced into the arm for the administration of contrast material during the test. Renal functions "creatinine level” were checked which must be normal (if GFR < 60 "normal”). Potential subjects were screened for intracranial clips, pacemakers, metal fragments or any other objects that would contraindicate MR imaging. All metallic objects were removed before going to scanner room. Patients were asked to change into hospital gown and last menstrual period (LMP) for pregnancy was checked.

Table 1. Age groping of the sample.

\begin{tabular}{ccc}
\hline Age group & Frequency & Percent \\
\hline Less than 35 & 10 & $9.5 \%$ \\
Between $35-55$ & 66 & $62.9 \%$ \\
More than 55 & 29 & $27.6 \%$ \\
Total & 105 & $100.0 \%$ \\
\hline
\end{tabular}




\subsection{Patient Position}

Patients were placed in prone position with head first and the breasts were placed directly in the center of each round opening of the $16 \mathrm{CH}$ breast array coil and head was placed at the head rest of the coil with the patient's arms at side or extended above the head.

\subsection{Patient Instruction}

Patient were given instructions not to move during the procedure, how to breath shallow and smoothly. Patients were informed that during the exam, the technologist is monitoring from the window and special camera and they will be able to talk to the technologist through microphone and they were asked to hold the emergency bottom in their hands if they needed any help during the exam. Then earplugs were placed to reduce the noise.

\subsection{Protocol Used}

The protocol used was shown in Table 2.

\subsection{Image Interpretation}

The images produced were independently evaluated using PACS workstations. MRI images of each patient were reviewed with computer aided analysis for the presence of any malignancy and the radiologist makes comments on points include: the background parenchymal enhancement pattern of the breast, type of lesion (foci, mass \& non-mass like), lesion morphology characteristic e.g. shape "round, oval, lobulated or irregular", margin "smooth, irregular or speculated", internal enhancement and distribution. Also, the lesion location is defined, size and kinetic curve characteristic. Associated findings are mentioned either, nipple retraction, skin thickening, edema, cyst and any abnormality of lymph nodes. Post-contrast high-resolution imaging is required to visualize important morphological information if focal enhancement is present. Then, in conclusion the assessments and recommendation is written as:

*Assessments; negative, benign, probably benign, suspicious or highly suggestive of malignancy.

*Recommendation; second look by US or MG, follow up, short interval f/u or for biopsy.

\subsection{Data Analysis}

Demographic patients' data and medical history were collected which includes hormonal medications, family history of breast disease, family history of other cancers, the phase of menstrual cycle and MRI screening findings. The raw data was tabulated then statistically analyzed with the use of SPSS program for Windows, version 19.0.0.

\section{Results}

Between Jan, 2012 and Dec, 2012, 280 women were included as a study sample. But 175 patients were excluded from the analysis for the following reasons: symptomatic patients, the contrast-enhanced MRI not completed, results were not available, biopsy not performed, biopsy results were not available or MRI result was not certain and they recommend ultrasound for better assessments. Thus, data from the 280 women was reduced to 105 (37.5\%) cases only. $41 \%$ of the patients did not perform a mammogram because they were recommended for direct MRI screening or follow up, while 59\% had a mammogram with positive result and referred to MRI for further evaluation (Figure 1).

\subsection{MRI Result}

The whole sample had performed MRI and the results were: $67.6 \%$ had a benign result $(-$ ve), $17.1 \%$ uncertain MRI result (?) and $15.2 \%$ the result was malignant (+ve). Accord to age grouping the highest malignant results were on women above 55 y/o and highest benign result on women between 35 - 55 y/o (Figure 2).

\subsection{Biopsy Findings}

Out of 105 patients, only 48 patients had performed biopsy under mammographic and ultrasound guidance. 
54.3\% did not perform biopsy, 32.4\% had biopsy with benign result and 13.3\% had biopsy but with malignant result. The highest benign and malignant result is at the age between 35 - 55 y/o (Figure 3).

Table 2. Protocol used with the parameters.

\begin{tabular}{|c|c|c|c|c|c|c|c|c|c|}
\hline Plane & Image series & $\mathrm{TR}$ & $\mathrm{TE}$ & IT & FA & NEX & SL & Matrix & FOV \\
\hline & localizer & 7.6 & 3.53 & - & 20 & 2 & 6 & 256 & 400 \\
\hline Tra & T2-tse-2mm & 3000 & 89 & - & 80 & 1 & 2 & 320 & 400 \\
\hline Tra & T1-tse-2mm & 590 & 15 & - & 80 & 1 & 2 & 320 & 400 \\
\hline \multirow[t]{2}{*}{ Tra } & T2-tirm-2mm & 4060 & 52 & 230 & 70 & 2 & 2 & 256 & 400 \\
\hline & Diffusion-spair & 5000 & 87 & - & - & 3 & 4 & 192 & 400 \\
\hline pause & \multicolumn{9}{|c|}{ Contrast injection } \\
\hline Tra & T1-fl3d-pre dyn FS & 4.55 & 1.61 & - & 10 & 1 & 2 & 480 & 400 \\
\hline \multirow[t]{3}{*}{ Tra } & T1-fl3d-post dyn FS & 4.55 & 1.61 & - & 10 & 1 & 2 & 480 & 400 \\
\hline & svs-se-breast-ref & 2000 & 100 & - & 90 & 1 & - & - & - \\
\hline & Svs-se-breast & 1500 & 100 & - & 90 & 128 & - & - & - \\
\hline
\end{tabular}

Abbreviation: TR, repetition time; TE, echo time; IT, inversion time; FA, flip angle; NEX, number of excitations; SL, slice thickness; FOV, field of view; C.M., contrast media; Tra, transverse; tse, turbo spin echo; tirm, turbo inversion recovery magnitude; dyn, dynamic; FS, fat saturation; svs, single voxel spectroscopy; se, spin echo; ref, reference.

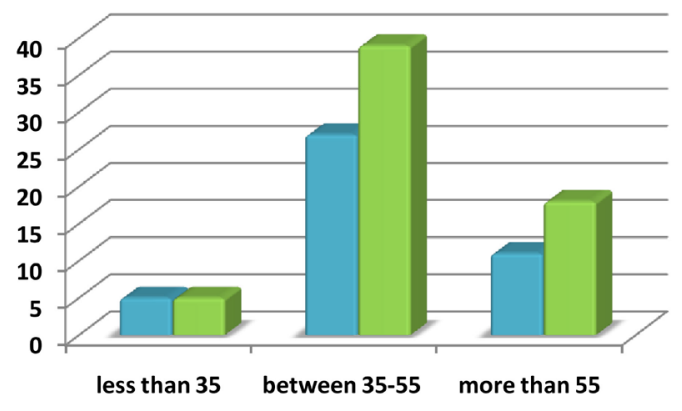

not performed

$\square$ performed with positive result

Figure 1. The distribution of mammogram result according to age grouping.

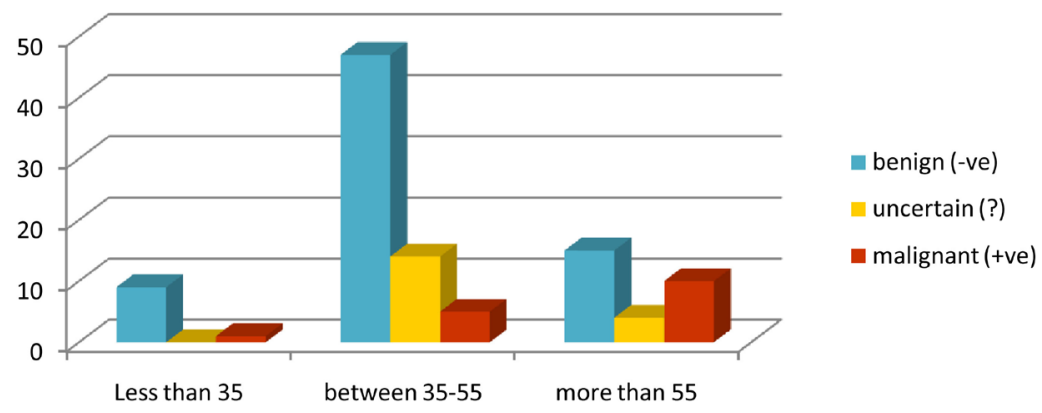

Figure 2. The distribution of MRI result according totype of neoplasm.

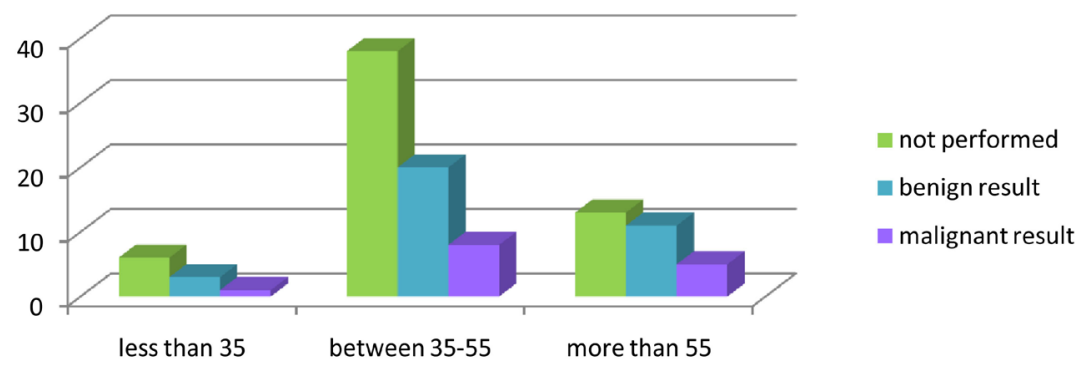

Figure 3. The distribution of biopsy result according to age. 


\subsection{Correlations between the Family History and the MRI Result}

The distribution of the sample according to family history, 71 patients did not have a family history and 34 patients had a family history as shown in Table 3 while the correlations between the personal history and the MRI result indicated that the personal history risk factor was more common than the family history as shown in Table 4. About $44.76 \%$ of the sample had different types of personal history (Figure 4), while only $32.38 \%$ had a family history. The total patients who had a personal history were 47 patients out of 105, and 58 patients did not have a personal history. $45 \%$ had cancer history on the left breast and $39 \%$ on the right breast. This means that $84 \%$ had breast cancer before while the other $16 \%$ had other different types of personal history risk factor.

\subsection{MRI Sensitivity and Specificity}

Attribution to biopsy to be as a gold standard to detect the Sensitivity and Specificity of the MRI result. A total 48 patients only of 105 were included in this study, those who performed biopsy, to compare their biopsy result with the MRI result to detect how the Sensitivity and Specificity of MRI is From Table 5. The false-negative (FN) results of MRI were 5 patients out of 14 positive result patients (35.7\% FN) so, 9 patients were detected as positive result by MRI and biopsy confirmed that. And the false-positive (FP) results were 11 patients out of 34 negative result patients (32.3\% FP), this means that the other 23 patients were assigned as negative result (benign) by MRI and the biopsy confirmed benignity of the lesion.

Calculation has been done to know the following the result: Sensitivity of MRI was $64.28 \%$ (true positive is highly detected) Specificity of MRI was $67.64 \%$ (true negative is higher) Positive predictive value (PPV) $45 \%$. Means if the MRI result was positive, the patient has $45 \%$ chance of actually positive result. Negative predictive value (NPV) $82.18 \%$. Means if MRI result was negative, then the patient has $82.18 \%$ a chance of not having breast cancer.

\subsection{Most Common MRI Findings}

The prevalence of different breast diseases detected by MRI for the whole sample was shown in Figure 5 . Intraductal carcinoma it is also called Ductal carcinoma in situ is the most common malignant (non-invasive) disease as the prevalence in (Figure 5) shows. It composes about $10.47 \%$ of MRI findings. This type of cancer develops within milk ducts of the breast. One of the most benign findings was fibrocystic changes. Almost 25.71\% of the patients had a fibrocystic disease. The following images show breast cancer (Figure 6) Ductal carcinoma in situ and invasive ductal carcinoma was diagnosed in 55 years old female and in a 69 years old female patient with family history. They proved to be DCIS and IDC after biopsy had been done. $4.86 \%$ of MRI findings were invasive ductal carcinoma which is the most common (invasive) malignant disease. While multiple masses of the right breast was seen in a 65 years old female patient (Figures 7-9).

\section{Discussion}

Mammograms are less likely to find breast tumors in women younger than 50 years than in older women. This is

Table 3. Correlation of MRI results with Family History.

\begin{tabular}{rrcccc}
\hline & & & MRI results & Total \\
\cline { 3 - 5 } & & Benign (-ve) & Not Sure (?) & Malignant (+ve) & \\
\hline \multirow{2}{*}{ Family History } & No & 44 & 13 & 14 & 71 \\
& Yes & 27 & 5 & 2 & 34 \\
Total & & 71 & 18 & 16 & 105 \\
\hline
\end{tabular}

Table 4. Correlation of MRI results with Personal History.

\begin{tabular}{|c|c|c|c|c|c|}
\hline & & \multicolumn{3}{|c|}{ MRI results } & \multirow{2}{*}{ Total } \\
\hline & & Benign (-ve) & Not Sure (?) & Malignant (+ve) & \\
\hline \multirow{2}{*}{ Personal History } & No & 37 & 11 & 10 & 58 \\
\hline & Yes & 34 & 7 & 6 & 47 \\
\hline Total & & 71 & 18 & 16 & 105 \\
\hline
\end{tabular}


Table 5. Sensitivity and Specificity of MRI according to biopsy result.

\begin{tabular}{cccccc}
\hline & & Biopsy & & total \\
\hline \multirow{3}{*}{ MRI } & + & + & - & 20 \\
& - & 9 & 23 & 28 \\
& total & 5 & 34 & 48 \\
\hline
\end{tabular}

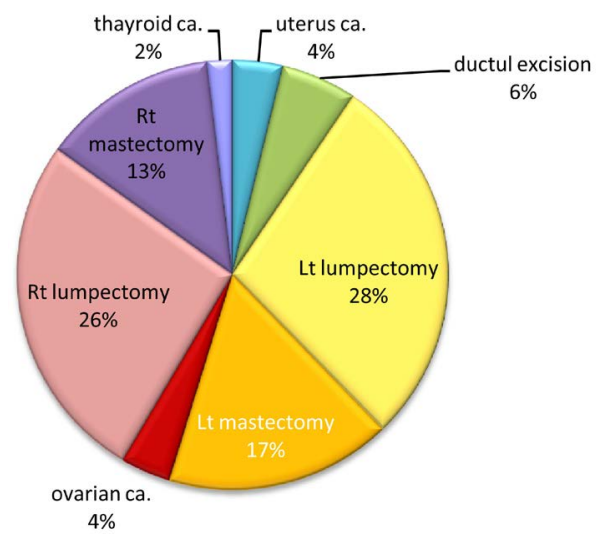

Figure 4. different types of personal history risk factor that the patients had.

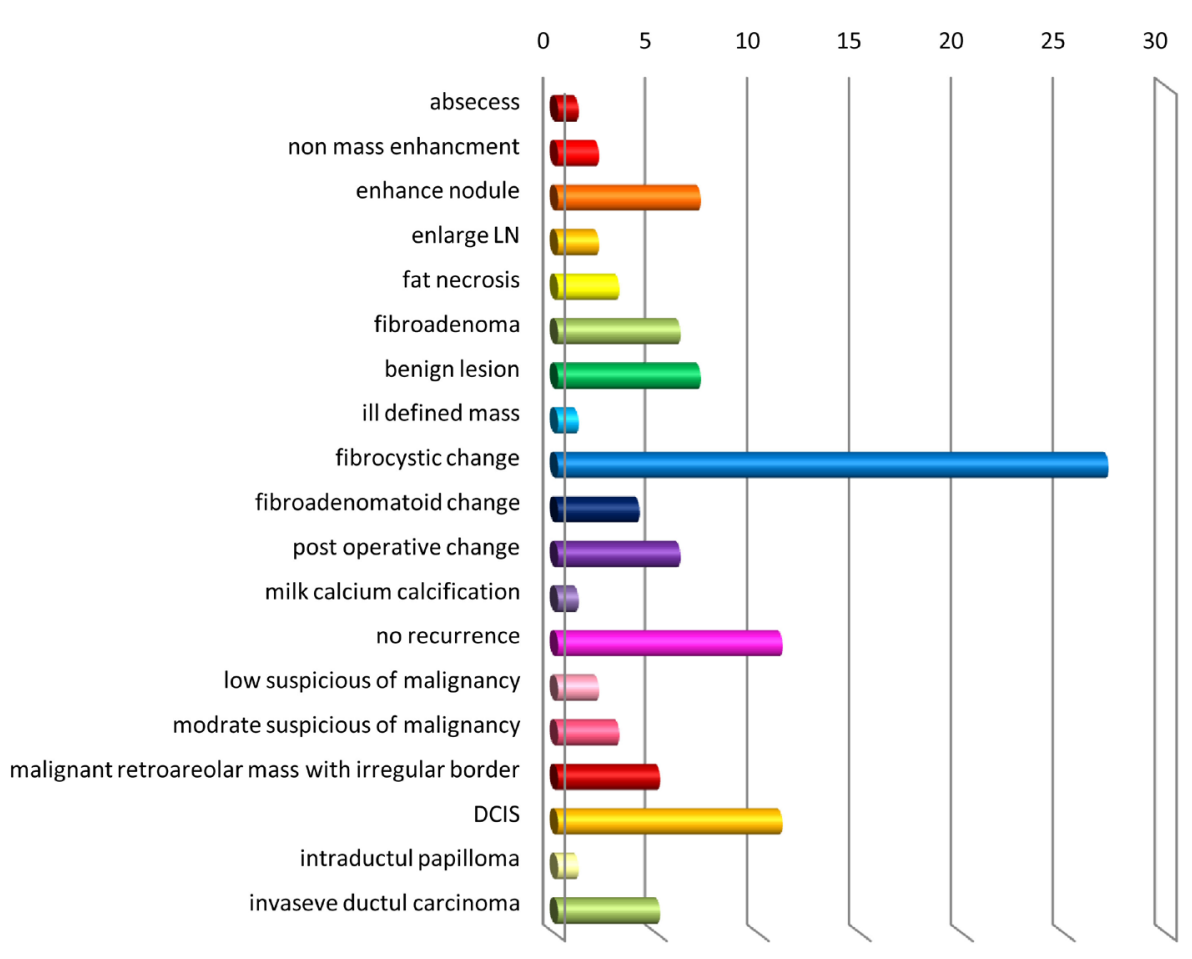

Figure 5. Breast disease finding..

due to the possibility that younger women have denser breast tissue that appears white on a mammogram. Because tumors also appear white on a mammogram, they can be harder to find when there is dense breast tissue [6]. Also MRI found cancer in the other breast that didn't show up yet on mammogram. In addition, after review of more recent studies, the American Cancer Society recently recommended that any woman with a greater than 

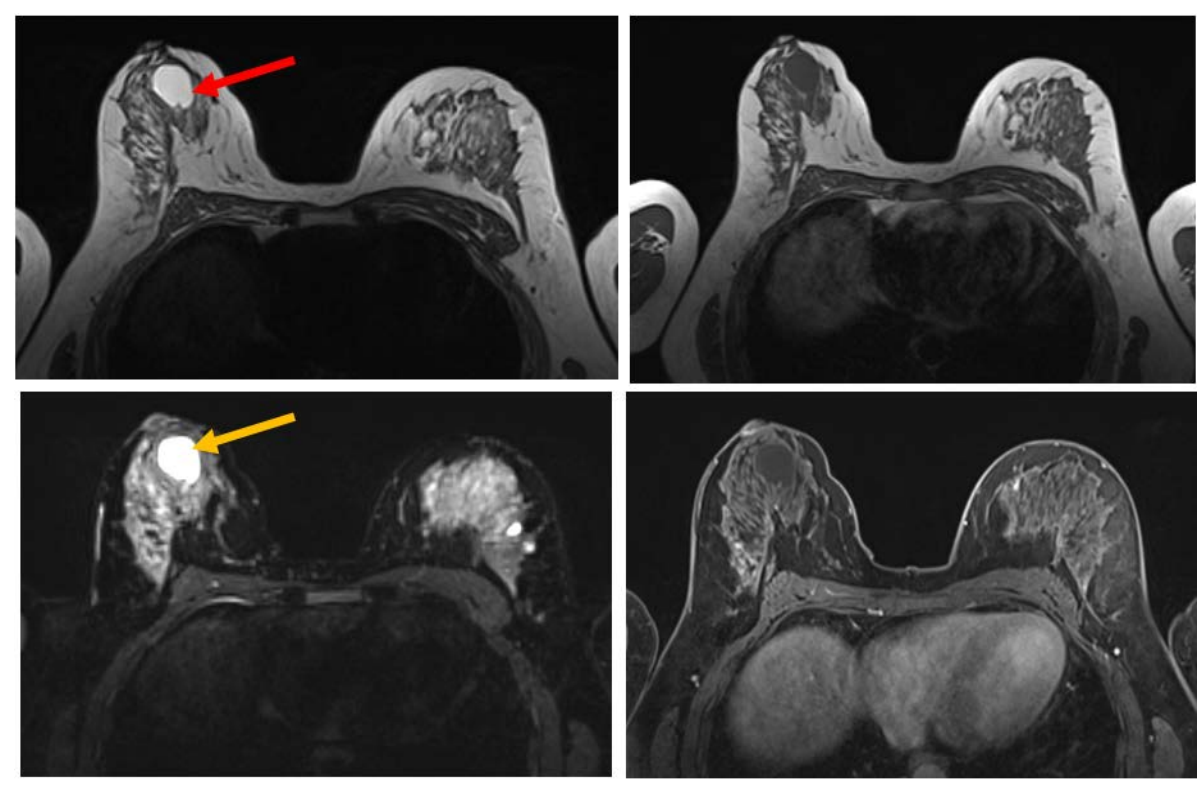

Figure 6. Axial MRI images. Both breast Show prominent glandular tissues with multiple bilateral cysts, the largest one in right breast retroareolarly, Appears as a round mass with smooth margins (well-defined) hyperintense in T2 and hypointense in T1 with very high signal on T2 tirm (fat-suppressed) image. After the injection of gadolinium it appears as hypointense _there is no enhancement_. tirm, turbo inversion recovery.

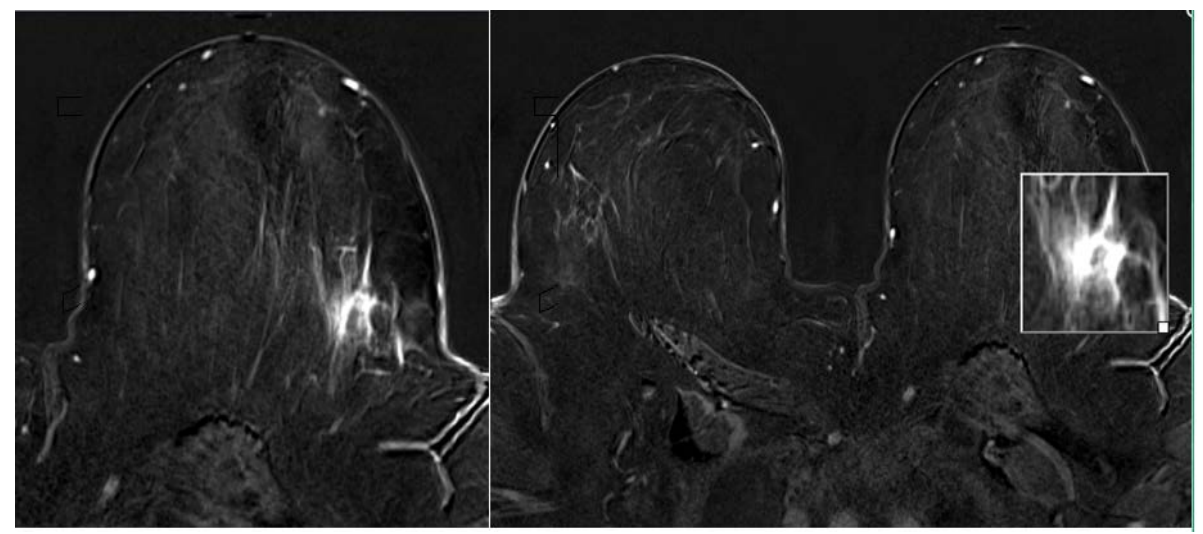

Figure 7. Axial MRI images T1 post contrast shows enlarge ducts in the left breast with high signal after gad injection. They are seen anterior and superior to the main lesion.

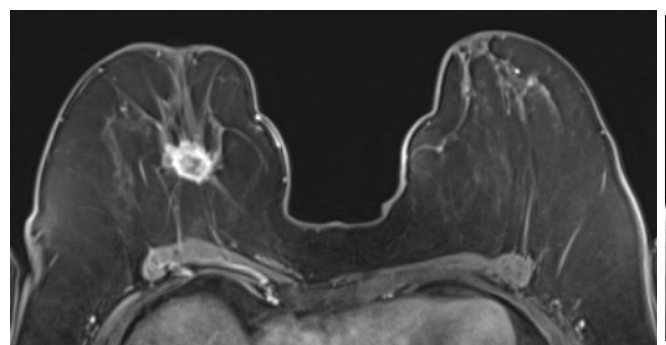

(a)

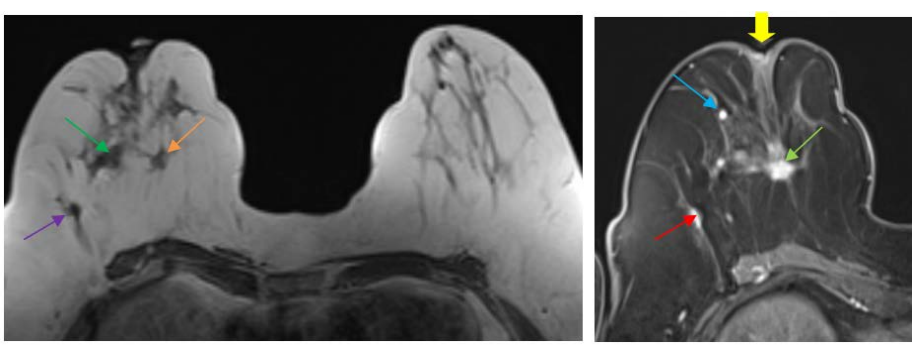

(b)

Figure 8. (a) Axial MRI T1 post contrast image. It shows a speculated mass enhancing in the right breast centrally. It measures $2 \times 1.3 \mathrm{~cm}$ in maximum dimension. This proved to be an invasive carcinoma. (b) Axial MRI images. There are multiple speculated peripherally enhancing nodules in the superior lateral aspect of the same breast (right). There is retraction of the nipple (Yellow arrow). 

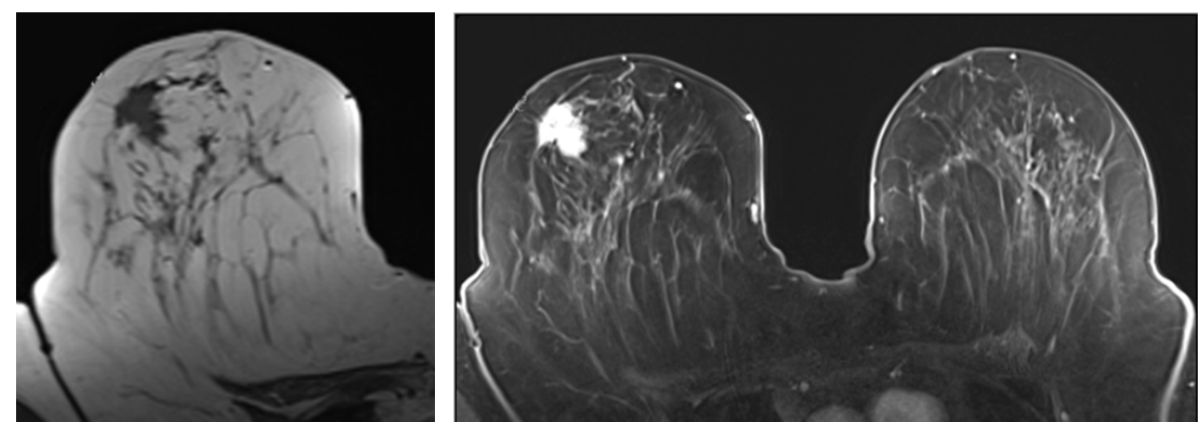

Figure 9. Axial MRI images. Large enhancing irregular mass is noted about $4.5 \mathrm{~cm}$ deep and lateral to the nipple, at 10 o'clock of the right breast. In its largest diameter the mass is measuring about $3 \times 2.7 \mathrm{~cm}$. it appears hypointense on T1 sequences and after gad injection there is an enhance. For definitive pathology biopsy has been suggested and it is proved to be invasive ductal carcinoma.

$20 \%$ - 25\% lifetime risk for breast cancer should consider undergoing screening with both mammography and breast MRI [7].

The addition of MRI examination of the mammogram in women at increased risk of breast cancer to detect the highest outcome for cancer but also was for false positive results to avoid the harm due to repeated testing or painful biopsies for those who do not have cancer. Therefore, it is advised to use MRI to examine women at high risk of breast cancer [8].

In our study, MRI findings of breast cancer is directly proportional to the age of the patient, where only $15.3 \%$ of the whole sample of their findings are malignancy, most of them $10.4 \%$ are more than 55 years, 3.8\% between 35 - 55 and $0.9 \%$ under 35 years. This association is also mentioned by [9]. While another study reported by [10] that is worthwhile to begin screening for women with a high risk from the age of 30 or 35 years old.

The percentage of women recommended by MRI for biopsy in this study is $45.7 \%$ and it is a very large percentage comparing to other studies i.e. [11] reporting only 2.9\% and [12] [13] reporting biopsy procedures with percentages of just over $15 \%$. Also, it was elsewhere mentioned [2] where the range was $6.3 \%$ similar to prior studies ranging (from 2.9\% to 15.8\%) among Screening Women at High Risk for Breast Cancer with Mammography and Magnetic Resonance Imaging.

Breast MRI is not recommended as a routine screening tool for all women. However, it is recommended for screening women who are at high risk for breast cancer, usually due to a strong family history and/or a mutation in genes such as BRCA1 or BRCA2. If you are considered high-risk, you would have breast MRI in addition to your annual mammograms [14].

Family history risk factor in our study composes $32.32 \%$, and from the result $1.91 \%$ of the cases have malignancy with positive family history. It is away different from prior studies' results and this is may be due to the large volume of the sample in those studies. In the present study, the personal history risk factor of breast cancer is found in $44.76 \%$ of the cases and only $5.7 \%$ are diagnosed with breast cancer with MRI and this is very similar to study for Berg W., et al., in Detection of Breast Cancer With Addition of Annual Screening Ultrasound or a Single Screening MRI to Mammography in Women With Elevated Breast Cancer Risk, where only (54\%) of participants had a personal history of breast cancer and (4.1\%) were diagnosed with cancer [6].

The sensitivity of MRI in our study is $64.28 \%$ and this is almost similar to prior studies (71\%) mentioned by [15]. In a study of [16] who reported that result of MRI sensitivity level ranging from $57 \%-100 \%$ due to differences in technical parameters such as the strength of the magnetic field, the type of coil and sequences used, the amount and timing of contrast media, the imaging and the reporting methods.

Specificity of breast MRI in our study is higher than the sensitivity of the MRI, our specificity result is 67.64\% also [15] reported MRI specificity value $89.8 \%$ which also higher than his sensitivity value, and this differences may be due to the difference in the size of sample.

Although this study is not a comparative study, but we can say that MRI has a better sensitivity than mammogram. From the results of mammography 59\% of the whole sample have a positive result, while MRI results say that only $15.2 \%$ of the sample have a positive result and after biopsy has performed the actual positive result is 
13.3\%. In a previous study [17], it was reported that MRI has high sensitivity ranging from $71 \%$ to $100 \%$ versus $16 \%$ to $40 \%$ for mammography in a high risk population. Screening mammograms alone, or screening breast ultrasounds alone, each found only a little more than half of the cancers. However, together, screening mammograms and ultrasounds found $82 \%$ of the cancers. Breast MRI (after three negative mammogram and ultrasound screenings) found another $8 \%$ of breast cancers not detected by the other tests.

In conclusion, from the study we found that the addition of magnetic resonance imaging to mammography in screening women at increased risk of breast cancer resulted in a higher cancer detection due to its high value of sensitivity.

\section{References}

[1] Lehman, C. (2006) Role of MRI in Screening Women at High Risk for Breast Cancer. Journal of Magnetic Resonance Imaging, 24, 964-970. http://dx.doi.org/10.1002/jmri.20752

[2] Lehman, C., Blume, J., Weatherall, P., Thickman, D., Hylton, N., Warner, E., et al. (2005) Screening Women at High Risk for Breast Cancer with Mammography and Magnetic Resonance Imaging. Cancer, 103, 1898-1905.

http://dx.doi.org/10.1002/cncr.20971

[3] Dummin, L.J., Cox, M. and Plant, L. (2007) Prediction of Breast Tumour Size by Mammography and Sonography-A Breast Screen Experience. The Breast Journal, 16, 38-46. http://dx.doi.org/10.1016/j.breast.2006.04.003

[4] Berg, W., Zhang, Z., Lehrer, D., Jong, R., Pisano, E., Barr, R., et al. (2012) Detection of Breast Cancer with Addition of Annual Screening Ultrasound of a Single Screening MRI to Mammography in Women with Elevated Breast Cancer Risk. JAMA, 307, 1394-1404. http://dx.doi.org/10.1001/jama.2012.388

[5] Gavenonis, S. (2011) Breast MR Imaging: Normal Anatomy. In: Liu, P.S., Ed., Normal MRI Anatomy from Head to Toe, an Issue of Magnetic Resonance Imaging Clinics, 1st Edition, Saunders Publisher, 519-507.

[6] Malur, S., Wurdinger, S., Moritz, A., Michels, W. and Schneide, R. (2001) A Comparison of Written Reports of Mammography, Sonography and Magnetic Resonance Mammography for Preoperative Evaluation of Breast Lesions, with Special Emphasis on Magnetic Resonance Mammography. Breast Cancer Research, 3, 55-60. http://dx.doi.org/10.1186/bcr271

[7] American Cancer Society (2013) What Are the Risk Factors for Breast Cancer? http://www.cancer.org/cancer/breastcancer/detailedguide/breast-cancer-risk-factors

[8] Afonso, N. (2009) Women at High Risk for Breast Cancer-What the Primary Care Provider Needs to Know. JABFM, 22, 43-50. http://dx.doi.org/10.3122/jabfm.2009.01.070188

[9] Sasieni, P., Shelton, J., Ormiston-smith, N., Thomson, C. and Silcocks, P. (2011) Among What Is the Life Time Risk o Developing Cancer? British Journal of Cancer, 11, 460-465.

[10] Urban, L. and Urban, C. (2012) Role of Mammography versus Magnetic Resonance Imaging for Breast Cancer Screening. Current Breast Cancer Reports, 4, 216-223 http://dx.doi.org/10.1007/s12609-012-0085-5

[11] Kriege, M., Brekelmans, C.T., Boetes, C., et al. (2004) Efficacy of MRI and Mammography for Breast-Cancer Screening in Women with a Familial or Genetic Predisposition. The New England Journal of Medicine, 351, 427-437. http://dx.doi.org/10.1056/NEJMoa031759

[12] Warner, E., Plewes, D.B., Hill, K.A., et al. (2004) Surveillance of BRCA1 and BRCA2 Mutation Carriers with Magnetic Resonance Imaging, Ultrasound, Mammography, and Clinical Breast Examination. JAMA, 292, 1317-1325. http://dx.doi.org/10.1001/jama.292.11.1317

[13] Morris, E., Liberman, L., Ballon, D.J., et al. (2003) MRI of Occult Breast Carcinoma in a High-Risk Population. AJR Am J Roentgenol, 181, 619-626. http://dx.doi.org/10.2214/ajr.181.3.1810619

[14] Orel, S.G. and Schnall, M.D. (2001) MR Imaging of the Breast for the Detection, Diagnosis, and Staging of Breast Cancer. Radiology, 220, 13-30. http://dx.doi.org/10.1148/radiology.220.1.r01jl3113

[15] Elmore, J., Armstrong, K., Lehman, C. and Fletcher, S. (2005) Screening for Breast Cancer. JAMA, 293, $1245-1256$. http://dx.doi.org/10.1001/jama.293.10.1245

[16] Forrai, G. (2011) Magnetic Resonance Imaging (MRI) in the Screening of High-Risk Patients and in the Detection and Diagnosis of Early Breast Cancer. In: Kahán, Z. and Tot, T., Eds., Breast Cancer, a Heterogeneous Disease Entity, Springer, 55-45. http://dx.doi.org/10.1007/978-94-007-0489-3_3

[17] Saslow, D., Boetes, C., Burke, W., Harms, S., Leach, M. and Lehman, C. (2007) American Cancer Society Guidelines for Breast Screening with MRI as an Adjunct to Mammography. CA: A Cancer Journal for Clinicians, 57, 75-89. http://dx.doi.org/10.3322/canjclin.57.2.75 
Scientific Research Publishing (SCIRP) is one of the largest Open Access journal publishers. It is currently publishing more than 200 open access, online, peer-reviewed journals covering a wide range of academic disciplines. SCIRP serves the worldwide academic communities and contributes to the progress and application of science with its publication.

Other selected journals from SCIRP are listed as below. Submit your manuscript to us via either submit@scirp.org or Online Submission Portal.
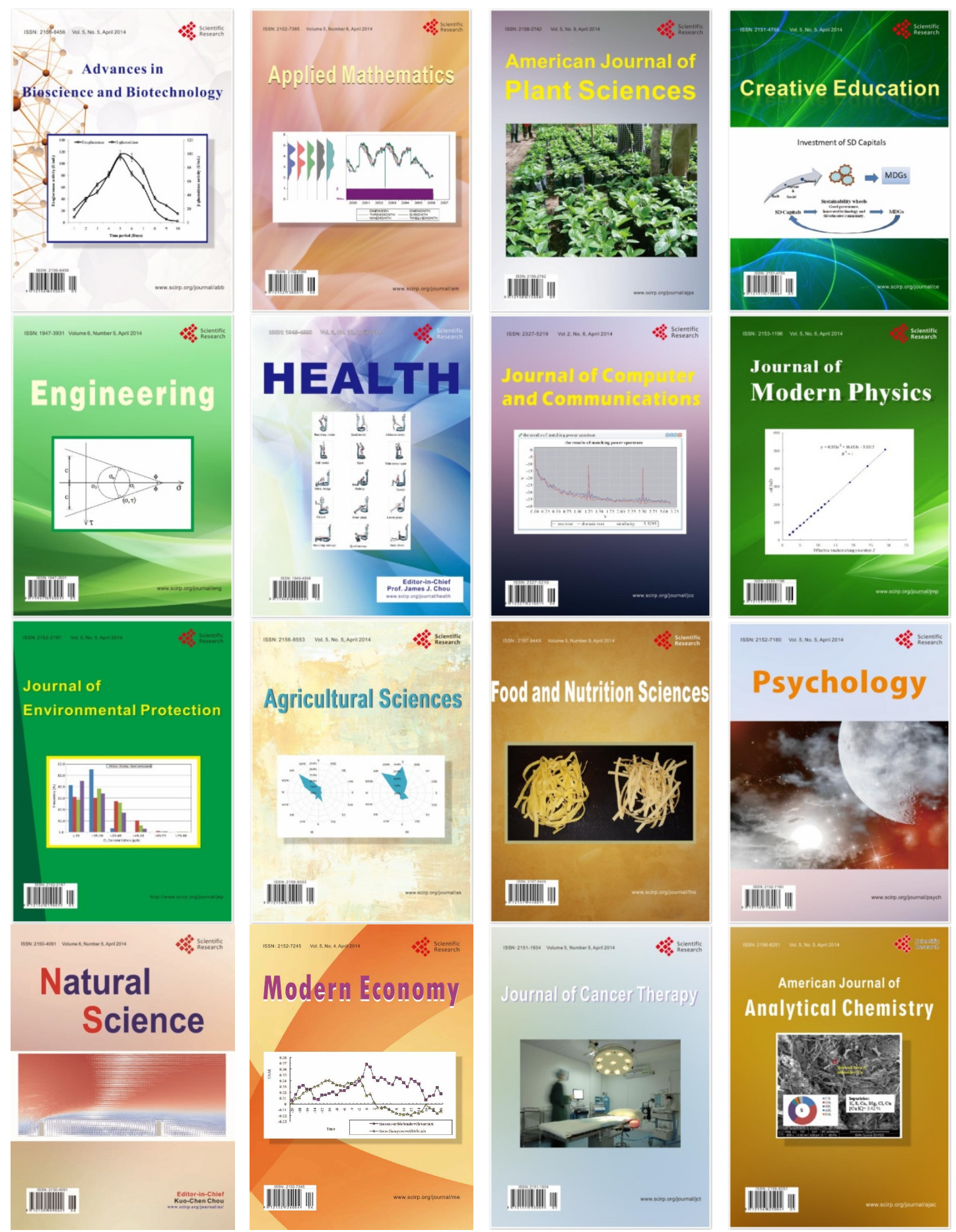